\title{
Grosse nationale Unterschiede
}

\section{Gerhard Kocher}

Dr. rer. pol., Gesundheitsökonom

Die Schweiz hat nach den USA das teuerste Gesundheitswesen, sowohl gemäss den Pro-Kopf-Ausgaben als auch in Bezug auf das Bruttoinlandprodukt. Einsame Spitze sind wir in der Direktbelastung der Patienten (Out-of-pocket-Zahlungen). Bezüglich Ärztedichte sind wir im 7. Rang von 34 Ländern. Die Spitalbettendichte ist ähnlich wie im OECD-Durchschnitt. Der Euro Health Index misst die Qualität der Gesundheitswesen. Im 1. Rang sind die Niederlande, gefolgt von der Schweiz.

Die Organisation für wirtschaftliche Zusammenarbeit und Entwicklung OECD in Paris hat ihre neuesten Zahlen zu den Gesundheitswesen der 37 OECD-Länder publiziert. Leider nicht mehr wie früher auf einer $C D$, sondern nur in ihrer Gesundheitsdatenbank, die im Internet zugänglich ist [1).

Die Gesundheitsstatistik enthält zahllose Daten zu Themen wie Lebenserwartung, Morbidität, Lebensstil, Gesundheitspersonal, Medizintechnik, Spitäler, Medikamente, Ausgaben und Finanzierung. Eine 17-seitige Liste führt alle Variablen auf [2]. Die Datenbank der OECD ist die umfangreichste internationale Datenquelle neben den Publikationen der WHO und der EU. Dieser Artikel wertet wie jedes Jahr verschiedene dieser Statistiken aus.

\section{Gesundheitsausgaben nach Sektoren: Abbau der Präventionsausgaben}

Gewisse Zahlenreihen der Datenbank zeigen von Jahr zu Jahr nur infinitesimale Zu- oder Abnahmen. Erst im Vergleich der letzten 10 oder 20 Jahre finden sich auffällige Veränderungen. Dazu gehört die Tabelle $1 \mathrm{mit}$ der Ausgabenentwicklung nach Sektoren. Das starke Kostenwachstum der Jahre 2005 bis 2009 wurde in den Jahren 2009 bis 2013 in allen sechs Sektoren jäh

Tabelle 1: Ausgabenwachstum nach Sektoren, OECD-Länder (in Prozent, real, pro Jahr und Kopf).

\begin{tabular}{lll} 
& $\mathbf{2 0 0 5 - 2 0 0 9}$ & $\mathbf{2 0 0 9 - 2 0 1 3}$ \\
\hline Stationäre Versorgung & 2,4 & 0,7 \\
\hline Ambulante Versorgung & 4 & 1,8 \\
\hline Langzeitpflege & 5,8 & 2,8 \\
\hline Medikamente & 1,9 & $-1,9$ \\
\hline Prävention & 5,6 & $-0,6$ \\
\hline Verwaltung & 3,8 & 0,9 \\
\hline
\end{tabular}

gebremst. Markant ist dies bei der Prävention: Das Wachstum von 5,6\% pro Jahr wurde abgelöst durch einen Rückgang von 0,6\% jährlich. Etwa die Hälfte der OECD-Staaten reduzierten seit 2009 die Präventionsausgaben, meist im Gegensatz zu den rhetorischen Lobreden über die Bedeutung der Prävention. Aber

Importantes différences nationales dans les dépenses de santé, la densité du personnel et des lits en hôpitaux

L'OCDE vient de publier ses dernières statistiques sur les systèmes de santé des 37 pays membres (www. oecd.org). A l'instar des années précédentes, le système de santé le plus cher reste celui des Etats-Unis. Avec 8713 \$ par personne et par année, les dépenses de santé sont en effet 1,52 fois plus élevées que la moyenne de I'OCDE (soit 5260 \$ de plus). La Suisse se classe à la deuxième place, avec $83 \%$ de plus que la moyenne. Avec $11,1 \%$ du produit intérieur brut, contre $8,9 \%$ pour la moyenne de I'OCDE, la Suisse se place au deuxième rang (avec les Pays-Bas), juste derrière les Etats-Unis. Dans tous les pays, les patients paient eux-mêmes une partie des coûts. Depuis plusieurs années, la Suisse est largement en tête du classement dans ce domaine, avec plus du double des Etats-Unis. En 2013, la densité médicale oscillait entre 1,8 et 6,3 médecins pour 1000 habitants. La Suisse occupe le $7^{\mathrm{e}}$ rang sur 34 pays et compte une densité médicale $21 \%$ plus élevée que la moyenne de I'OCDE. En revanche, le nombre de lits pour 1000 habitants se situe légèrement en-dessous de la moyenne de I'OCDE et se monte à 4,7 . La tête du classement est occupée par le Japon, la Corée du Sud et l'Allemagne. Chaque année, les pays de I'OCDE enregistrent 16194 sorties d'hôpital pour 100000 habitants, I'Autriche (64\% de plus) et l'Allemagne en tête. 
eben: Die Prävention ist die Zukunft der Medizin, sie war es schon immer und wird es immer bleiben ...

Bemerkenswert ist auch die Entwicklung des grössten Kostenblocks, der stationären Versorgung. Die jährliche Wachstumsrate zwischen 2005 und 2009 sank fast um einen Viertel auf 0,7\%. In etwa einem Dutzend OECD-Länder war sie ab 2009 sogar negativ.

\section{Ausgaben pro Kopf und in Prozent vom Bruttoinlandprodukt}

Die Tabelle 2 zeigt in der 2. Spalte die durchschnittlichen Gesundheitsausgaben 2013 pro Einwohner (kaufkraftbereinigt). Am teuersten ist wie seit Jahren das US-Gesundheitssystem, in dem pro Kopf und Jahr nicht weniger als 5260 USD mehr ausgegeben werden als im OECD-Durchschnitt. Die 8713 USD der USA sind $152 \%$ höher als die durchschnittlichen OECD-Ausgaben. Am zweitteuersten ist die Schweiz mit 83\% über dem Durchschnitt. Für 2015 werden die Schweizer Gesundheitsausgaben auf 75 Milliarden CHF geschätzt (9084 CHF pro Kopf), für 2016 auf 77,3 Milliarden CHF. Für die USA ist keine Besserung in Sicht. Nach den neuesten offiziellen Prognosen (Centers for Medicare \& Medicaid Services CMS, August 2015) werden die Ausgaben bis 2024 jährlich um 5,8\% steigen

Tabelle 2: Gesundheitsausgaben 2013, kaufkraftbereinigt

(Spalten 2-5: in USD/Kopf; Spalten 6-9: in \% des Bruttoinlandprodukts; Reihenfolge: gemäss 3. Spalte).

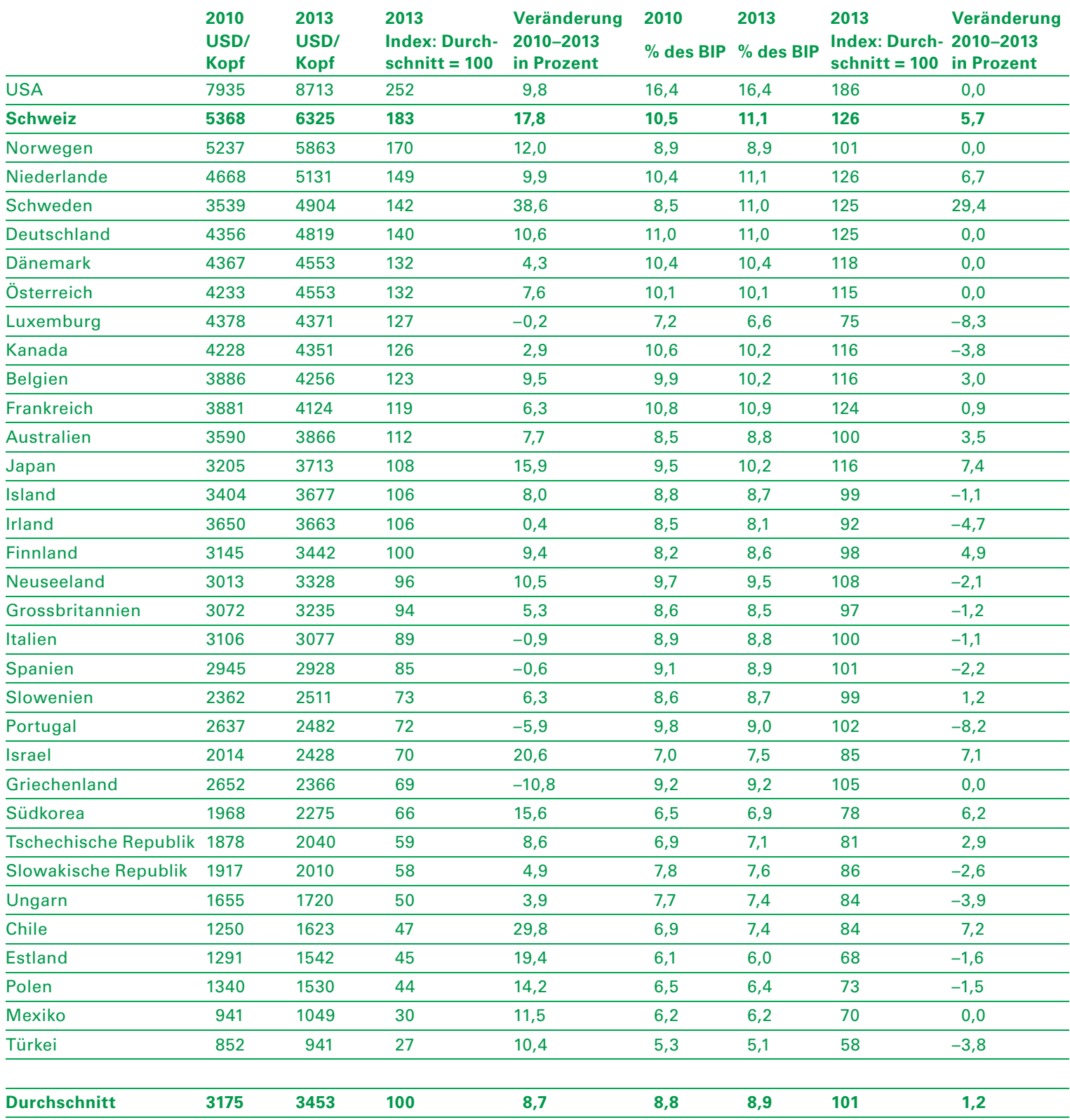


und damit 5,4 Billionen USD oder 19,6\% des Bruttoinlandprodukts erreichen.

Dass hohe Gesundheitsausgaben nicht eine hohe Qualität garantieren, ist für den Fall USA seit Jahrzehnten allgemein bekannt. Das trifft leider auch auf unser Land zu und wird in internationalen Qualitätsvergleichen immer deutlicher. Es gibt 13 Länder, die für eine gute oder sehr gute Gesundheitsversorgung sogar $25-50 \%$ weniger ausgeben als wir. Beispiele sind Dänemark, Österreich, Kanada, Belgien, Frankreich und Australien. Fünf weitere Länder mit guten Gesundheitssystemen zahlen immerhin 7-24\% weniger als wir. Auf die Schweiz 2015 umgerechnet, würden wir 5-16 Milliarden CHF jährlich einsparen. 2013 hätten wir mit den Pro-Kopf-Ausgaben Deutschlands 12 Milliarden USD gespart, mit den französischen Ausgaben 18 und mit den britischen Ausgaben 25 Milliarden USD.

Nicht nur ist unser Ausgabenniveau sehr hoch, sondern auch die Wachstumsrate der letzten Jahre. Von 2010 bis 2013 sind die durchschnittlichen Pro-KopfAusgaben der Schweiz um 17,8\% gestiegen. Dies ist das Doppelte des OECD-Durchschnitts von 8,7\% und sogar

Tabelle 3: Anteil der Selbstzahlungen am Total der Gesundheitsausgaben, 2013.

\begin{tabular}{|c|c|c|}
\hline & In Prozent & Index: Durchschnitt $=100$ \\
\hline Mexiko & 45,2 & 227 \\
\hline Südkorea & 35,9 & 180 \\
\hline Chile & 31,9 & 160 \\
\hline Griechenland & 28,4 & 143 \\
\hline Ungarn & 28,3 & 142 \\
\hline Schweiz & 26,0 & 131 \\
\hline Israel & 25,9 & 130 \\
\hline Polen & 22,7 & 114 \\
\hline Slowakische Republik & 22,4 & 113 \\
\hline Belgien & 20,4 & 103 \\
\hline Italien & 18,8 & 94 \\
\hline Finnland & 18,7 & 94 \\
\hline Estland & 18,2 & 91 \\
\hline Island & 18,1 & 91 \\
\hline Irland & 16,9 & 85 \\
\hline Schweden & 16,5 & 83 \\
\hline Türkei & 15,4 & 77 \\
\hline Kanada & 15,0 & 75 \\
\hline Tschechische Republik & 15,0 & 75 \\
\hline Deutschland & 13,0 & 65 \\
\hline Dänemark & 12,4 & 62 \\
\hline USA & 12,0 & 60 \\
\hline Slowenien & 11,9 & 60 \\
\hline Luxemburg & 11,0 & 55 \\
\hline Grossbritannien & 9,0 & 45 \\
\hline Frankreich & 7,5 & 38 \\
\hline Durchschnitt & 19,9 & 100 \\
\hline
\end{tabular}

\section{Selbstzahlungen}

In allen Ländern zahlen die Kranken einen Teil der Kosten direkt aus dem eigenen Geldbeutel. Dies sind die sog. Out-of-pocket-Zahlungen, deren Höhe von Land zu Land sehr verschieden sein kann (Tab. 3). Es sind Ausgaben für Pflegeheime, Institutionen für Behinderte, nicht kassenpflichtige Behandlungen, rezeptfreie Medikamente, Zahnmedizin, Brillen und Hörgeräte und Selbstbehalte. Weit an der Spitze ist wie seit Jahren die Schweiz, in der die Direktbelastung 3,5mal höher ist als in Frankreich. Sogar verglichen mit den USA sind die Selbstzahlungen bei uns mehr als doppelt so hoch.

Die Schweizer Bevölkerung muss pro Jahr 1630 USD (kaufkraftbereinigt) für Selbstzahlungen ausgeben 52\% mehr als die Amerikaner und 2,7-mal mehr als der OECD-Durchschnitt. Die Deutschen zahlen nur 649, die Franzosen 277 und die Niederländer 270 USD. Dass ausgerechnet die beiden Länder mit den weitaus höchsten Selbstzahlungen (Schweiz und USA) die teuersten Gesundheitswesen haben, deutet nicht gerade auf einen massiven Spareffekt der Selbstbeteiligungen hin. Die Höhe der Gesundheitsausgaben ist aber immer multifaktoriell bedingt. Monokausale Schlüsse sind den dümmeren unter den Gesundheitsökonomen, Politikern, Lobbyisten und Medien vorbehalten. 
Tabelle 4: Ärztedichte, 2013

(Ärzte pro 1000 Einwohner).

\begin{tabular}{|c|c|c|}
\hline Land & Ärzte & Index: Durchschnitt $=100$ \\
\hline Griechenland $^{1}$ & 6,3 & 191 \\
\hline Österreich & 5,0 & 152 \\
\hline Norwegen & 4,3 & 130 \\
\hline Portugal $^{2}$ & 4,3 & 130 \\
\hline Deutschland & 4,1 & 124 \\
\hline Schweden & 4,0 & 121 \\
\hline Schweiz & 4,0 & 121 \\
\hline Italien & 3,9 & 118 \\
\hline Spanien & 3,8 & 115 \\
\hline Tschechische Republik & 3,7 & 112 \\
\hline Dänemark & 3,6 & 109 \\
\hline Island & 3,6 & 109 \\
\hline Australien & 3,4 & 103 \\
\hline Israel & 3,4 & 103 \\
\hline Slowakische Republik ${ }^{1}$ & 3,4 & 103 \\
\hline Estland & 3,3 & 100 \\
\hline Frankreich $^{1}$ & 3,3 & 100 \\
\hline Niederlande $^{1}$ & 3,3 & 100 \\
\hline Ungarn & 3,2 & 97 \\
\hline Belgien & 3,0 & 91 \\
\hline Finnland & 3,0 & 91 \\
\hline Luxemburg & 2,8 & 85 \\
\hline Neuseeland & 2,8 & 85 \\
\hline Grossbritannien & 2,8 & 85 \\
\hline Irland & 2,7 & 82 \\
\hline Kanada $^{1}$ & 2,6 & 79 \\
\hline Slowenien & 2,6 & 79 \\
\hline USA & 2,6 & 79 \\
\hline Japan & 2,3 & 70 \\
\hline Südkorea & 2,2 & 67 \\
\hline Mexiko & 2,2 & 67 \\
\hline Polen & 2,2 & 67 \\
\hline Chile $^{2}$ & 1,9 & 58 \\
\hline Türkei $^{1}$ & 1,8 & 55 \\
\hline Durchschnitt & 3,3 & 99 \\
\hline
\end{tabular}

${ }^{1}$ Praktizierende Ärzte plus andere, die im Gesundheitswesen als Manager, Lehrende, Forscher usw. tätig sind. Dies erhöht gemäss OECD die Ärztedichte um 5-10\%

${ }^{2}$ Alle Ärzte mit Lizenz zur Berufsausübung.

\section{Schweizer Ärztedichte über dem Durchschnitt}

Gemäss Tabelle 4 variierte die Ärztedichte 2013 von 1,8 bis $6,3(1: 3,5)$ pro 1000 Einwohner, die Pflegendendichte von 1,8 bis $17,4(1: 9,7)$. Weltweit sind die Unterschiede laut WHO [4] viel grösser: Ärztedichte von 0,01 bis 7,7 ( $1: 770)$, die Pflegendendichte von 1,2 bis 8,0 $(1: 6,7)$. Bei der Ärztedichte ist die Schweiz im 7. Rang von 34 Ländern und 21\% höher als der OECD-Durchschnitt. Viel weniger Ärzte pro 1000 Einwohner haben unter anderem Grossbritannien, Kanada und die USA.

\section{Schweizer Bettendichte ähnlich wie der OECD-Durchschnitt}

Die Bettenzahl pro 1000 Einwohner liegt in der Schweiz mit 4,7 leicht unter dem OECD-Durchschnitt von 4,9. Die höchste Bettendichte haben Japan, Südkorea, Deutschland und Österreich. Seit 2010 nahm die Bettenzahl in 21 Ländern ab, in 7 blieb sie unverändert und in 3 Ländern stieg sie (Durchschnitt: minus 0,1 Bett). Für die 20 Jahre bis 2010 liegen die Zahlen für 17 Länder vor. Insgesamt nahm die Bettendichte um 37\% ab. Die Gründe sind zahlreich: Strukturreformen der stationären Versorgung, Änderungen bei den Tarifen, Sparmassnahmen, medizinische Fortschritte, Rückgang der durchschnittlichen Aufenthaltstage bei vielen Eingriffen, Verlagerung von Kranken in die ambulante Versorgung und Ausbau der Spitex.

\section{Krankenhausaustritte 2013 als Indikator für die Spitallastigkeit}

Jährlich werden pro 100000 Einwohner im Schnitt 16194 Spitalaustritte gezählt. An der Spitze der 31 Länder liegen Österreich (64\% höher) und Deutschland (56\% höher). In der Schweiz sind es nur 5 Prozent mehr als der Durchschnitt. Damit ist sie im 11. Rang von 29 Ländern. Als Krankenhausaustritte definiert die OECD die Zahl der Patienten, die nach stationärer Behandlung und mindestens einer Nacht im Spital ausgetreten oder gestorben sind.

\section{Weitere Ländervergleiche}

- Nützliche Vergleiche bietet das Buch International Profiles of Health Care Systems [5]. Der renommierte Commonwealth Fund in New York stellt darin die Gesundheitssysteme von 15 Ländern auf je 10 Seiten dar. Autor des Kapitels über die Schweiz ist Paul Camenzind vom Obsan in Neuenburg. 2012 erschien ein ähnliches Werk: Institutionelle Charakteristiken der Gesundheitssysteme: Eine Studie über 29 OECDLänder [6].

- Der New Yorker Internetdienst für Finanz- und Wirtschaftsinformationen 24/7 Wall St. publizierte eine Weltrangliste der "gesündesten und der ungesündesten Länder» [7]. Sie basiert auf $21 \mathrm{Kri}$ terien zu den Themen 1. Gesundheitszustand/ Lebenserwartung/Lebensstil, 2. Zugang zu sauberem Wasser, sauberer Luft und Elektrizität sowie Ärztedichte und 3. Pro-Kopf-Gesundheitsausgaben, Armut und Arbeitslosigkeit. Die nach diesen Kriterien gesündesten zehn Länder von insgesamt 172 sind Katar, Norwegen, Schweiz, Luxemburg, 
Japan, Island, Österreich, Singapur, Schweden und Australien.

- Eine Studie [8] im Auftrag der EU versucht, den Einfluss von drei Lebensstilfaktoren (Alkohol, Tabak und Übergewicht) auf die Effizienz des Gesundheitssystems $\mathrm{zu}$ eruieren. Erfasst sind 28 EU-Staaten sowie Island und Norwegen. Trotz Einsatz verschie-

\section{Dass ausgerechnet die beiden Länder mit den} höchsten Selbstzahlungen die teuersten Gesundheitswesen haben, deutet nicht gerade auf einen Spareffekt der Selbstbeteiligungen hin.

denster Methoden lässt sich kein Einfluss der drei Faktoren auf den Effizienzvergleich nachweisen. Dies gilt auch für den Vergleich der nationalen Gesundheitsausgaben, wo andere Faktoren eine viel grössere Rolle spielen.

- Das Gesundheitswesen von 37 europäischen Ländern vergleicht der Euro Health Consumer Index 2014 [9]. Für jedes Land werden 48 Indikatoren bewertet. Wie schon im Vorjahr siegen die Niederlande mit 898 Punkten vor der Schweiz mit 855 Punkten. Es folgen Norwegen, Finnland und Dänemark. Deutschland ist im 9., Österreich im 10., Frankreich im 11. und Grossbritannien im 14. Rang. In nicht weniger als vier der sechs Themenbereiche belegen die Niederlande den 1. Rang. Die Schweiz siegt bezüglich Zugang zur Gesundheitsversorgung und Wartezeiten. Herausgeberin des aufschlussreichen 101-seitigen Berichts ist das schwedische Health Consumer Powerhouse.

Korrespondenz: Dr. rer. pol. Gerhard Kocher Haldenweg $10 \mathrm{~A}$ CH-3074 Muri
Im November 2015 wird die OECD die wichtigsten Statistiken aus ihrer Datenbank in einer Broschüre Health at a glance 2015 publizieren. Auf diesen Termin hin werden zudem die aktualisierten OECD-Qualitätsindikatoren [11] der nationalen Gesundheitssysteme veröffentlicht. Diese Qualitätsvergleiche nehmen erfreulicherweise immer mehr zu. Vergleiche des Inputs und der Ausgaben müssen durch Qualitätsdaten ergänzt werden. Dem Niveau der gesundheitspolitischen Diskussion kann dies nur nützen.

Literatur

1 www.oecd.org/els/health-systems/health-data.htm

2 www.oecd.org/els/health-systems/List-of-variables-OECD-HealthStatistics-2015.pdf

3 Konjunkturforschungsstelle KOF/ETH, Frühjahrsprognose der schweizerischen Gesundheitsausgaben 2013-2016. Zürich; 2015. www.kof.ethz.ch/de/publikationen/p/kof-studien/3743/

4 World Health Statistics 2015. 164 Seiten, WHO Geneva, 2015 www.who.int/gho/publications/world_health_statistics/2015/en

5 International Profiles of Health Care Systems, The Commonwealth Fund, New York, 2015.164 S. Volltext: www.commonwealthfund. org/ /media/files/publications/fund-report/2015/jan/1802 mossialos intl profiles 2014 v7.pdf?la=en

6 Valérie Paris et al. Verlag SGGP, Bern, 120 Seiten. Auch in Französisch. www.sggp.ch

7 Thomas C. Frohlich et al. The Healthiest (and Least Healthy) Countries in the World, 24/7 Wall St. 2015. http://247wallst.com/ special-report/2015/04/03/the-healthiest-and-least-healthycountries-in-the-world/2/

8 Comparative efficiency of health systems, corrected for selected lifestyle factors. European Commission, 2015. 156 Seiten. http:// ec.europa.eu/health/systems_performance assessment/ docs/2015_maceli_report_en.pdf

9 Euro Health Consumer Index 2014, Stockholm. www.healthpowerhouse.com/files/EHCI_2014/EHCI_2014 report.pdf

10 International comparisons of healthcare quality. The Health Foundation/Nuffield Trust. London; 2015. www.quality watch.org. uk/sites/files/qualitywatch/field/field_document/QualityWatch_ International comparisons full report.pdf

$11 \mathrm{http} / / /$ stats.oecd.org/Index.aspx? 